\title{
Initial Design and Testing of Multiplayer Cooperative Game to Support Physical Activity in Schools
}

\author{
Solomon Sunday Oyelere ${ }^{1, * \mathbb{C}}$, Simon Malmström Berghem ${ }^{1}$, Robert Brännström ${ }^{1}$, Stina Rutberg ${ }^{2}{ }^{\mathbb{D}}$, \\ Teemu H. Laine ${ }^{3}$ and Anna-Karin Lindqvist ${ }^{2}$ \\ 1 Department of Computer Science, Electrical and Space Engineering, Luleå University of Technology, \\ 93187 Luleå, Sweden; bersim-8@student.ltu.se (S.M.B.); robert.brannstrom@ltu.se (R.B.) \\ 2 Department of Health, Education and Technology, Luleå University of Technology, 97187 Luleå, Sweden; \\ stina.rutberg@ltu.se (S.R.); Anna-Karin.Lindqvist@ltu.se (A.-K.L.) \\ 3 Department of Digital Media, Ajou University, Suwon 16499, Korea; teemu@ubilife.net \\ * Correspondence: solomon.oyelere@ltu.se
}

Citation: Oyelere, S.S.;

Berghem, S.M.; Brännström, R.;

Rutberg, S.; Laine, T.H.;

Lindqvist, A.-K. Initial Design and Testing of Multiplayer Cooperative Game to Support Physical Activity in Schools. Educ. Sci. 2022, 12, 100.

https://doi.org/10.3390/

educsci12020100

Academic Editors: Lazar Stošić, Łukasz Tomczyk, Alla Belousova and Laura Fedeli

Received: 24 November 2021

Accepted: 25 January 2022

Published: 31 January 2022

Publisher's Note: MDPI stays neutral with regard to jurisdictional claims in published maps and institutional affiliations.

Copyright: (c) 2022 by the authors. Licensee MDPI, Basel, Switzerland. This article is an open access article distributed under the terms and conditions of the Creative Commons Attribution (CC BY) license (https:// creativecommons.org/licenses/by/ $4.0 /)$.

\begin{abstract}
Recent studies have shown that children are not adequately physically active and there is a need to increase children's physical activity. This study describes new opportunities and solutions for using existing games and gamification to increase physical activity among children in Sweden. We adopted the principles of Tic-Tac-Training to redesign, build, and test a classical multiplayer cooperative game, Battleship, to create a PA game that children experience as fun and engaging. The low fidelity prototype of the game was developed using an iterative game development life cycle and tested with 13 young male children aged 8-11 in a real-world informal setting. A mixed-method research approach was used to understand the users' experiences and the impact of the Battleship-PA game on behavior change regarding physical activity. Research data were collected through audio recordings of interactions, direct observation, and a user experience questionnaire. The results of this study indicate both positive and negative feedback that can be used to improve the game and user experiences. The results from the unfiltered recordings revealed that both teams were competitive, cooperated within their team, and became excited whenever they destroyed opponent's ships or were close to winning. However, the children felt bored and exhausted when many gamification tasks were repeated several times in a game session. Direct observation indicated that the children enjoyed the physical activities resulting from playing the game. However, participants who had not previously played the classical version of Battleship were confused about the objectives and concept of the game. The analysis of the user experience questionnaire indicated that most children found the game easy to play, motivating, engaging, interactive, fun, cooperative, competitive, and visually appealing. Furthermore, most children agreed that the game helped them to be physically active and strongly agreed that they enjoyed performing the physical activities in the game. Future work is needed to improve the game user interface, gamification elements, and prepare additional physical activity tasks for a rewarding experience.
\end{abstract}

Keywords: gamification; multiplayer cooperative game; physical activity; active school transport; formative evaluation; game architecture

\section{Background}

\subsection{Physical Activity}

The World Health Organization (WHO) has recommended children and adolescents be physically active at least an average of 60 min per day and should engage in intense physical activity at least three days per week [1]. However, most children and adolescents do not reach these recommendations [2] and miss out on the health benefits of physical activity. These benefits include cardiorespiratory and muscular fitness as well as positive changes in weight [2]. In general, a healthy lifestyle with continuous adequate physical activity decreases the chances of cancer, diabetes, and cardiovascular diseases $[3,4]$. 
The WHO ranks physical inactivity as the fourth leading cause of non-communicable diseases and argues that physical inactivity is a global pandemic causing major human suffering and is responsible for a substantial economic burden in societies worldwide [5]. Other data indicate that physical activity has a positive effect on cognitive abilities and prosocial behavior [6]. Furthermore, research has shown that healthy habits involving physical activity and a healthy lifestyle are more easily adopted during childhood or adolescence and are often retained into adulthood [7]. Therefore, improving daily physical activity habits is a priority of the WHO's global plan for increasing physical activity [8].

\subsection{Gamification as a Solution for Increased Physical Activity}

Excessive screen time has been suggested to be a contributing factor to childhood inactivity. However, research also recognizes that gamification has great potential for children's motivation and learning. For example, uptake of the augmented reality game Pokémon Go has been unprecedented, and the game has motivated players to be more physically active. Gamification is defined as the use of game elements (e.g., playing rules, scoring, leaderboards, badges, etc.) in non-gaming contexts. Gamification can be used to motivate and engage individuals to perform activities that they normally would avoid, such as physical activity. Research has shown that games are strong motivators that can drive people to perform unappealing activities [9], such as cycling on snowy winter roads, [10] and using active transport (AT) instead of motorized transport [11]. Several gamification frameworks have been proposed to guide researchers and developers into using gamification to solve various problems, including Octalysis [12], MDA [13], and Hexad [14].

To promote and motivate healthier and more physically active lifestyles, recent studies have suggested using gamification to increase physical activity among schoolchildren and adolescents. Patricio, Aires, and Medeiros [15] found that gamification could motivate overweight adolescents to increase their physical activity in school settings, including testing with 65 adolescents aged 15-19. Gamification increased participants' adherence to weight loss programs after a four-week period. Another Irish study [16] involved a 24-week intervention to observe if gamification could change adolescents' behavior regarding physical activity. This intervention was designed for team and individual competitions using motivation incentives and was shown to change adolescent behavior towards physical activity.

Besides, previous study has shown that applying gamification elements in health and fitness applications increased the potential to influence and engage people in regular physical activity [17]. Gamification-based physical activity intervention was used to analyze the differences between motivational affordances of gamification and feedback elements and provided insights into design guidelines for incorporating motivational elements according to different age groups in game-based technology [18].

Previous study [19] evaluated the usability and effectiveness of the gamification application that promotes physical activity and found that there were no significant differences between a quantified version of the application and the gamified version which offers virtual reward and social comparison. The findings from [19] shows the need for further research and reflection to facilitate the understanding of important aspects of gamified physical activity to enable the design and development of new ubiquitous game elements. Furthermore, additional research, such as this study, will develop deeper understanding into the motivational affordances of gamification in physical activity in informal education contexts. Regarding users' experiences in gamification solutions for physical activity, previous studies have shown that there was a decrease in enjoyment and usefulness of gamification elements with continuous use, which shows that users are prone to the novelty effect of gamification application [20].

Gamification and game-based learning approaches have been applied in training programs to prevent obesity in children between 8-12 in age in informal educational 
settings [21]. A highly satisfactory outcome was obtained for biometric variables, learning healthy habits, and user experience [21].

An international study [22] of 3817 children aged 9-13 from 12 different schools from England and Canada explored if a four-week walking competition could increase the number of students walking to school. Most of the students reported that walking to school made them feel happier, healthier, made more friends, had more time with their friends, and could more easily concentrate in class. Julien et al. [23] developed an educational collaborative game where students reported their daily physical activity during physical education class, recess, and transport home. Their activity was then compared to running distances using the same amount of energy as the students had used while active. The study revealed that teachers wanted activities in the game tied to the school and that it is possible to deploy PA solutions in public schools.

\section{Study Scope, Research Question and Contribution}

This study is inspired by the game Tic-Tac-Training [24] to promote sustainable behavior concerning adult active transportation. Tic-Tac-Training is a variation of the classical game Tic-Tac-Toe; here, players collaborate in teams competing against other teams, instead of having two players as in the classical game. The gameboard in Tic-Tac-Training has several sizes, and all of them are much larger than the classical $3 \times 3$ tile game board. To claim tiles, users perform a task related to PA or healthy living that could be customized, thus allowing the system to be used in several contexts. Battleship-PA was inspired to allow hosts to customize tasks and players performing tasks before claiming a tile as well as the system architecture. Tic-Tac-Training was designed for competition among multiple teams and provided some drive to players to continue gameplay over multiple matches.

An earlier study showed that the competitive approach was not satisfactory or wellsuited for children because they largely desire cooperation, teamwork, and collaboration over competition [25]. Tic-Tac-Training was designed and developed for adults in workplaces, but children have distinct and peculiar viewpoints about games and what motivates them in interesting games. Therefore, it becomes important to reconsider the game development approaches and seek better ways to encourage children to be physically active. The novelty of this study is focused mainly on adapting an existing classical game, Battleship, to allow students and teachers to create tasks that suit their orientation of PA. Previous studies have shown the importance of the active involvement of end-users in the design and development of digital technologies - especially technologies that focus on behavior change [24]. Moreover, the opportunity for teachers to create their own tasks in the game, e.g., from the curriculum, makes it more attractive to use the game in school contexts when aiming to increase child PA. There is also a need to consider other focus areas of the game and technology development to address the needs of society, such as health, wellbeing, and climate action by factoring in the expectation and needs of end-users.

The end-user's experience should be applied in the creation of gamification experience and digital solutions for behavior change [24]. As the first step in game adaptation, we created a digital version of the Battleship game to understand if it would be playable by the students. In a previous intervention in a school setting, we aimed to increase physical activity in the form of active school transportation. Assignments designed by teachers and connected to the curriculum were used as gamification elements to increase the children's motivation for active school transport as well as increasing teachers' engagement $[26,27]$. Therefore, in the design phase of the Battleship-PA, we wanted to create a game that allowed teachers to create assignments that integrate learning activities into the game while also increasing PA. To understand how PA can be promoted in formal, informal, and non-formal school settings through gamification, we applied here an iterative game development life cycle. This model transforms the classical strategy 2-player Battleship game into a multiplayer PA game, Battleship-PA, that features cooperative, teamwork, and collaboration elements. Using a mixed-method research approach, we evaluated the first prototype of the Battleship-PA to determine user experience and impact. 
The specific research question guiding this study is, what is the user experience and impact of the Battleship-PA game to promote motivation for physical activity among school-aged children in an informal setting?

This study is part of a multidisciplinary project "Sustainable Innovations for Children Transporting Actively (SICTA).". Among other things, the SICTA project aims to develop novel ideas and ways to apply gamification to increase children's PA and to engage in active school transport.

\subsection{Our Contributions: Initial Design, Development and Implementation of Multiplayer Cooperative Game, Battleship-PA, to Promote Active School Transport}

\subsubsection{Initiation}

A low fidelity prototype of the Battleship game was developed using an iterative game development life cycle (GDLC) [28] and inspired by the Tic-Tac-Training game [24]. The GDLC presented in Figure 1 consists of six stages: initiation, pre-production, production, testing, beta, and release.

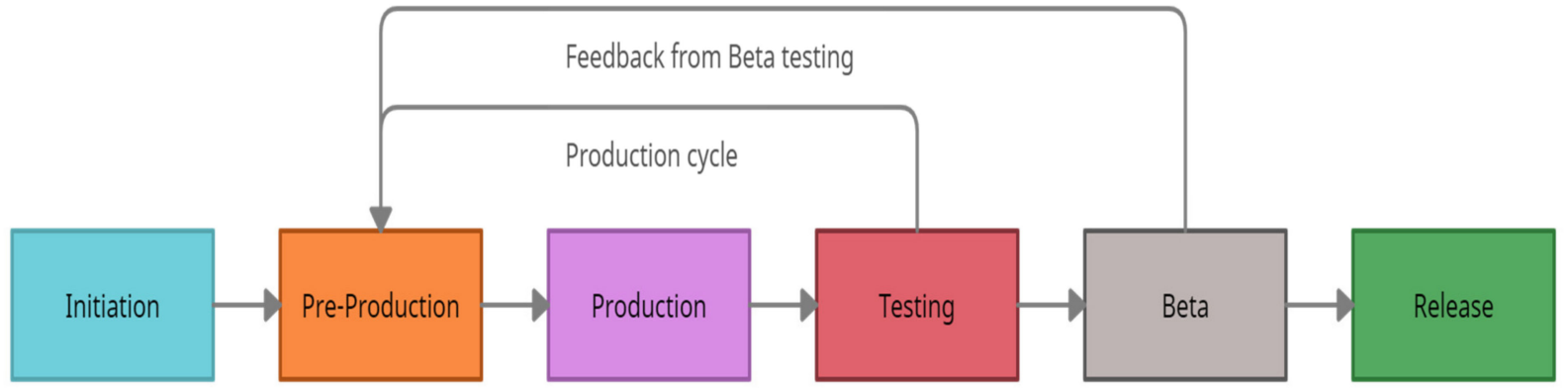

Figure 1. Battleship-PA game development life cycle.

The battleship game was chosen because of its closely related features with the TicTac-Toe game used in the previous study and that inspired this study. Similar to the Tic-Tac-Toe game, Battleship is a two-player strategy game that supports cooperation, collaboration, teamwork, and competition. Both games can be tweaked and implemented as a multiplayer game. One unique feature of the Battleship game that makes it appealing for this intervention is the large size of the game board $(10 \times 10$ grid). More tasks can be generated during gameplay with a higher number of grids. These tasks are PA-oriented and are meant to allow users to perform PA while playing the game. The Tic-Tac-Training game presented in [24] was targeted at adults in workplaces.

Initiation describes the initial rough idea of the game, pre-production defines the idea, and production is the core development of the idea and testing consisting of users tested the developed version. After the testing stage evaluates the game, the process goes back to pre-production with insights gained from the testing stage. If the game is deemed to be sufficiently refined after the testing stage, then the development process proceeds into the beta testing stage, thus letting third party users or external testers play the game; the game is then released to the public.

\subsubsection{Pre-Production}

This study presents the iterative game development process of Battleship-PA followed by gameplay, testing, and formative evaluation in an informal school setting. Finally, we apply the Octalysis framework [12] to analyze the implemented features of Battleship-PA, including how it connects to the eight core drives of gamification, to examine whether it has the potential to change children's behavior around PA in the future. The lessons learned here synthesize the experiences acquired from the entire process and provide valuable information not only for game developers, but also for other stakeholders that use persuasive game technology for behavioral changes in children regarding their PA. 


\subsubsection{Production: Basic Gameplay of Battleship-PA}

This game is a web-based variation of the classical game "Battleship." The goal is to encourage schoolchildren's PA. Battleship-PA gives the players a cooperative and competitive experience that extends the basic concepts from the classical version of Battleships. In the classical version, two players first place their ships on their own board and then take turns guessing where the opponent's ships are placed on their board. They gain points by correctly guessing their opponent's ship's placements. The match ends when one player has found all the other player's ships.

Battleship-PA uses a web-based version. It can have several players, and both teams' ships are placed randomly on their board or may be adjacent to other ships. Figure 2a shows the game board when newly created. Teams still score by correctly guessing the other team's ships positions. The team must complete a physical task in order to guess. We consider the process of completing the physical task, following certain rules as gamification. The process of performing the physical activity (i.e., gamifying the activity) while playing the Battleship game is the primary source of gamification in the current version of the prototype application. The assignments that the teacher/instructor provided in the game during the physical activity is the idea for gamification, mixing learning experiences with physical activity in a gamification setting, for example, looking for traffic signs on their way to school, or counting the number of steps on their way. All of the current and future gamification elements are presented in the discussion section. We intend to use the evaluation results and feedback to add additional gamification features. Before deciding which tile to guess, teams can hover or click the tile to discover the task description, to understand how to perform the task. Figure $2 \mathrm{~b}$ presents the task popup example, and Table 1 shows some examples of physical activity tasks that are gamified in the current version of Battleship-PA. Reading is crucial, as the board simply shows an icon that might not convey the task's requirements. Other tasks might have the same icon; therefore, reading is important to differentiate tasks with the same icon. The teams do not have to take turns, which suggests that one team can guess several times before the other team can guess. Therefore, completing tasks faster than the other team is a valid strategy to win the match. The process is performed similarly to the classical version - correctly guessing all the other team's ships. The ships are formed by guessing the correct tiles, which will be indicated with a green color. Figure $2 c$ shows the difference between ships, misses, and unclaimed tiles. Figure $2 \mathrm{~d}$ presents the winning message, e.g., a case where Team 1 has won.

Table 1. Physical activity tasks that were gamified and implemented in the game for the team and individual playing the Battle-PA game.

\begin{tabular}{cc}
\hline Team Tasks & Individual Tasks \\
\hline Spin around 5 times & Spin around 5 times \\
Touch the door as fast as you can & Touch the door as fast as you can \\
Touch your feet or as low down as you can & Win rock paper scissors \\
Stand on one leg for $15 \mathrm{~s}$ (right/left) & Win rock paper scissors 3 times in a row \\
Make a line in height order & Touch your feet \\
Make a line in age order & Stand up without using your hands \\
Make numbers 1-3 with your bodies & Stand on one leg for $15 \mathrm{~s}$ (right/left) \\
Take a lap around the room & Take a lap around the room \\
\hline
\end{tabular}




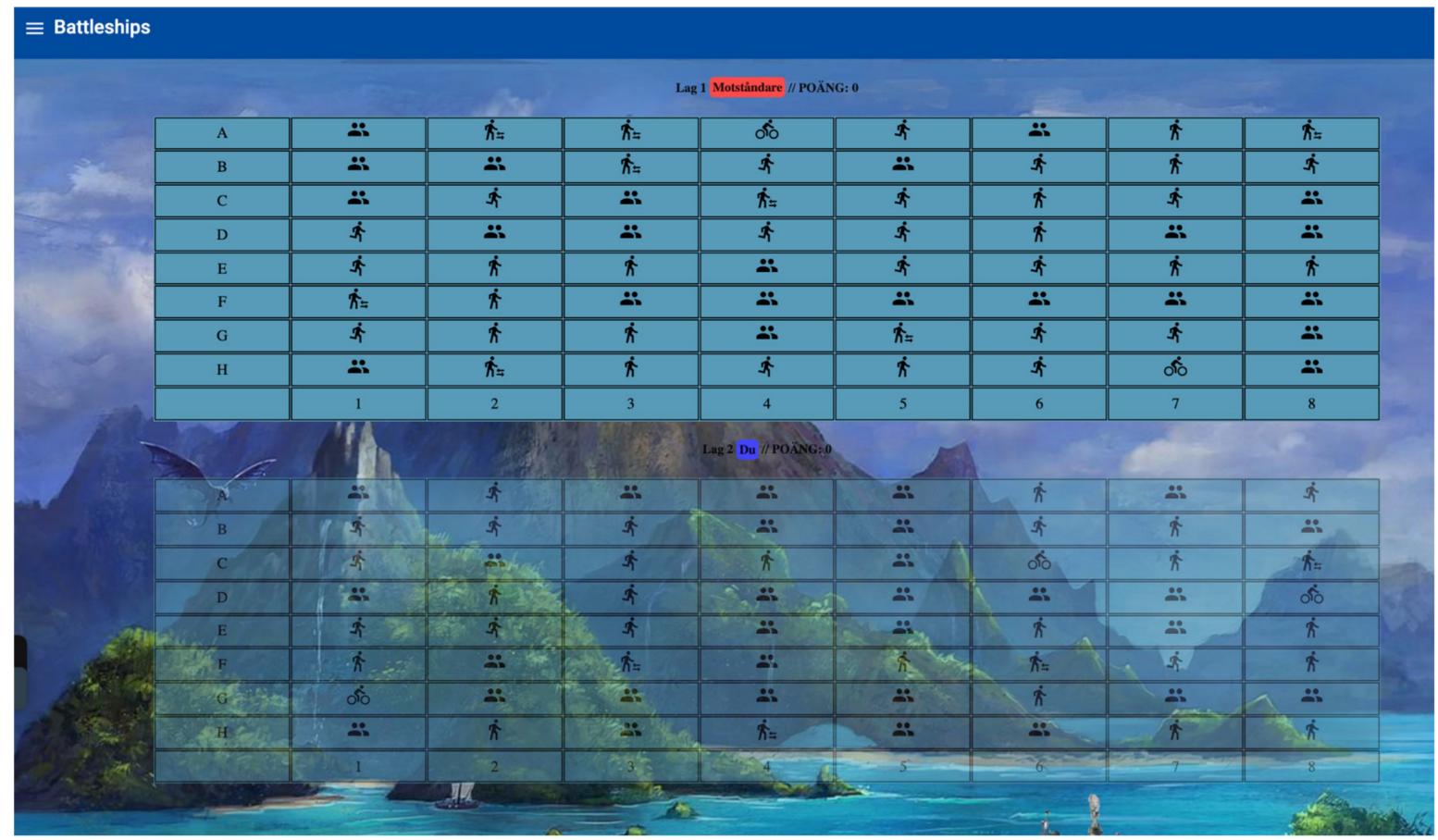

(a)

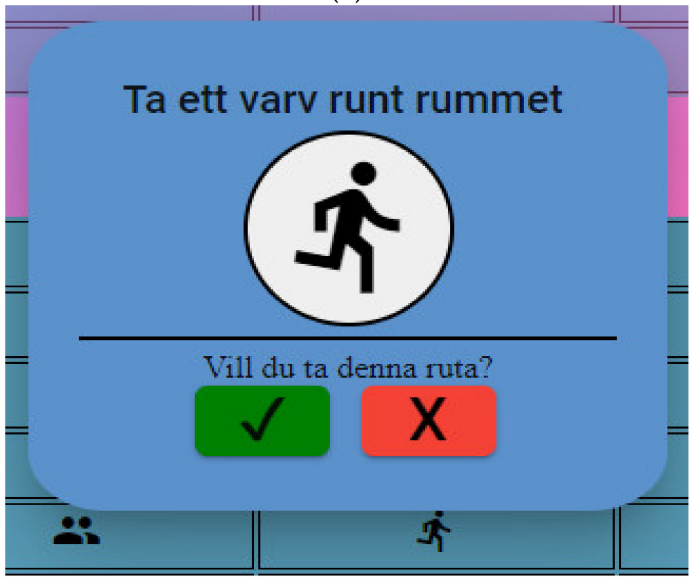

(b)

\begin{tabular}{|c|c|c|}
\hline$\ddot{~}$ & $\dot{x}$ & $\dot{x}=$ \\
\hline$\overline{\dot{i}_{F}}$ & 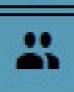 & $\overline{\bar{x}}$ \\
\hline $\bar{\phi}$ & $\bar{\phi}$ & $\overline{\bar{x}}$ \\
\hline $\bar{i}$ & $\vec{i}$ & $\dot{t}$ \\
\hline$\ddot{\circ}$ & $\ddot{8}$ & $\dot{R}=$ \\
\hline$\dot{\phi}$ & $\ddot{8}$ & $\dot{\phi}$ \\
\hline $\bar{i}$ & $\overline{i t}$ & $\overline{\bar{t} \dot{\phi}}$ \\
\hline
\end{tabular}

(c)

Figure 2. Cont. 


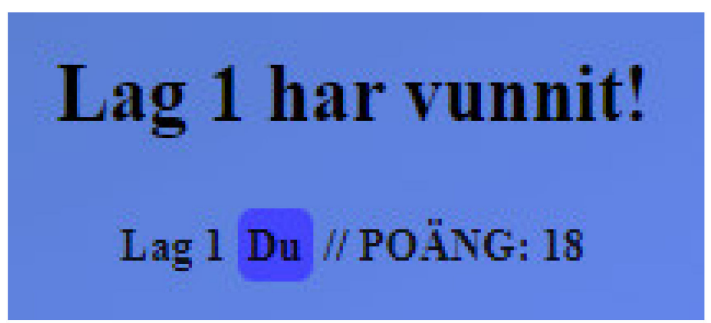

(d)

Figure 2. (a) Board when newly created. (b) Task popup example. Upper text: "Take a lap around the room", lower text: "Do you want to take this tile?". (c) Difference between ships (green tiles), misses (red tiles), and unclaimed (cadet blue tiles). (d) Winning message: Team 1 has won! Team 1 (You)//Points: 18.

\subsubsection{Match and Task Creation}

The welcoming screen presents an interface for users to log in (Figure 3a) and have a view of the current state of the game (Figure $3 b$ ). Creating a Battleships-ST match is simple: All users can create matches by choosing the create match option in the navigation bar (Figure 3c) and taking the host role for that particular match. This will create a lobby with a six-character code for joining the game, which consists of capital letters and randomized numbers; the backend ensures that the code is unique. Users then enter the code in the join match option in the navbar and then join one of the two teams in the lobby. They can then switch teams before the match has begun (Figure 3d). The host has an option to proceed from the team lobby to create tasks for the match while other users are still in the lobby. A task is made up of a description and an icon. The description is written by the host, and an icon is then chosen from a dropdown menu and then submitted. The icon should be linked to the description so that users can easily categorize tasks by simply observing the board. For example, for a task with the description "bike to school", a bike icon would be most fitting since the task involves biking. Submitted tasks are placed in the task list and can be removed by the host if they wish by clicking the trashcan button (Figure 3e). The host can proceed whenever they decide that enough tasks have been added. They can then start the match, and the board will then be populated with randomly selected tasks from the task list. Since the selection is completely random, some tasks might appear more frequently than other tasks, and there is a chance that some tasks do not appear at all.

\subsubsection{Architecture and Implementation}

Battleship follows a client-server architecture model that web-based clients can access with a web browser. Figure 4 illustrates the game architecture implemented with Angular, NodeJS, Sequelize ORM, and MariaDB technologies. The frontend architecture splits features into several angular services that are accessed using the user interface (UI). The components are related to the gameboard, e.g., uses board (for general gameplay), team, and match services (for general game variables). Similarly, the match creation components utilize task, match, auth, and team creation services. The login components use the auth service to allow logins and creation of users. The Angular services then communicate with the backend managers using a REST API. The backend consists of several Sequelize managers that perform various database actions to handle data on users, boards, teams, matches, etc. 

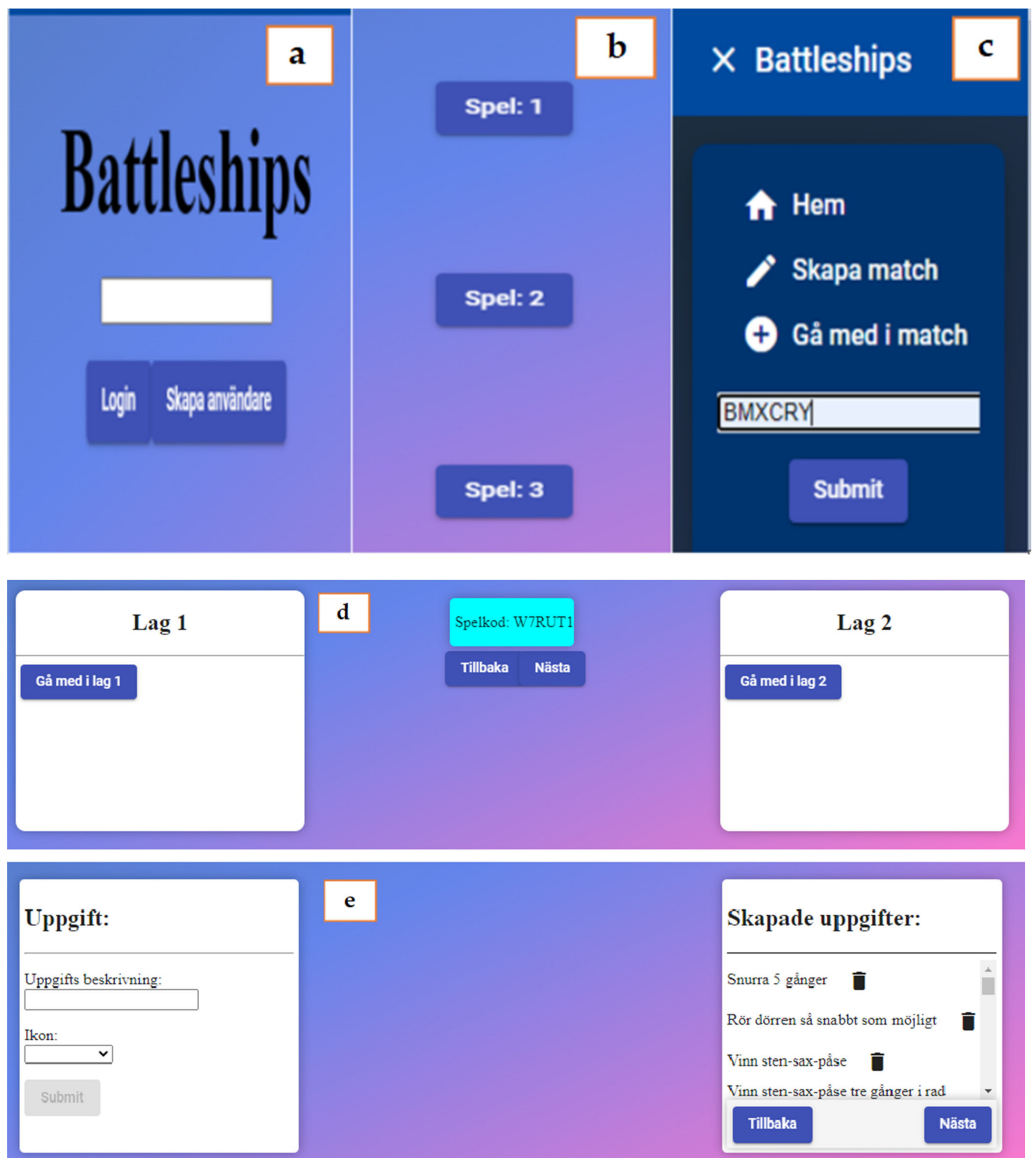

\section{e}

\section{Skapade uppgifter:}

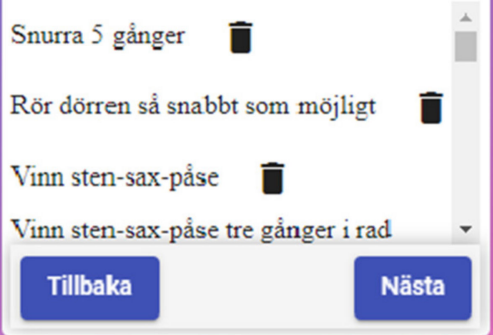

Figure 3. (a) Login screen. Lower buttons, login and create user. (b) Overview screen, game: [gameID]. (c) Navigation bar with buttons-home, create match and join match. (d) Team lobby. Left menu-Task, task description, icon. Right side-created tasks, spin 5 times, touch the door as fast as possible, win rockpaper-scissors, win rock-paper-scissors three times in a row. Lower buttons on the right side-back, next. (e). Task creation, Team 1 and Team 2 with options join Team 1/2. Middle part game code: WTRUT1, back and next buttons. 


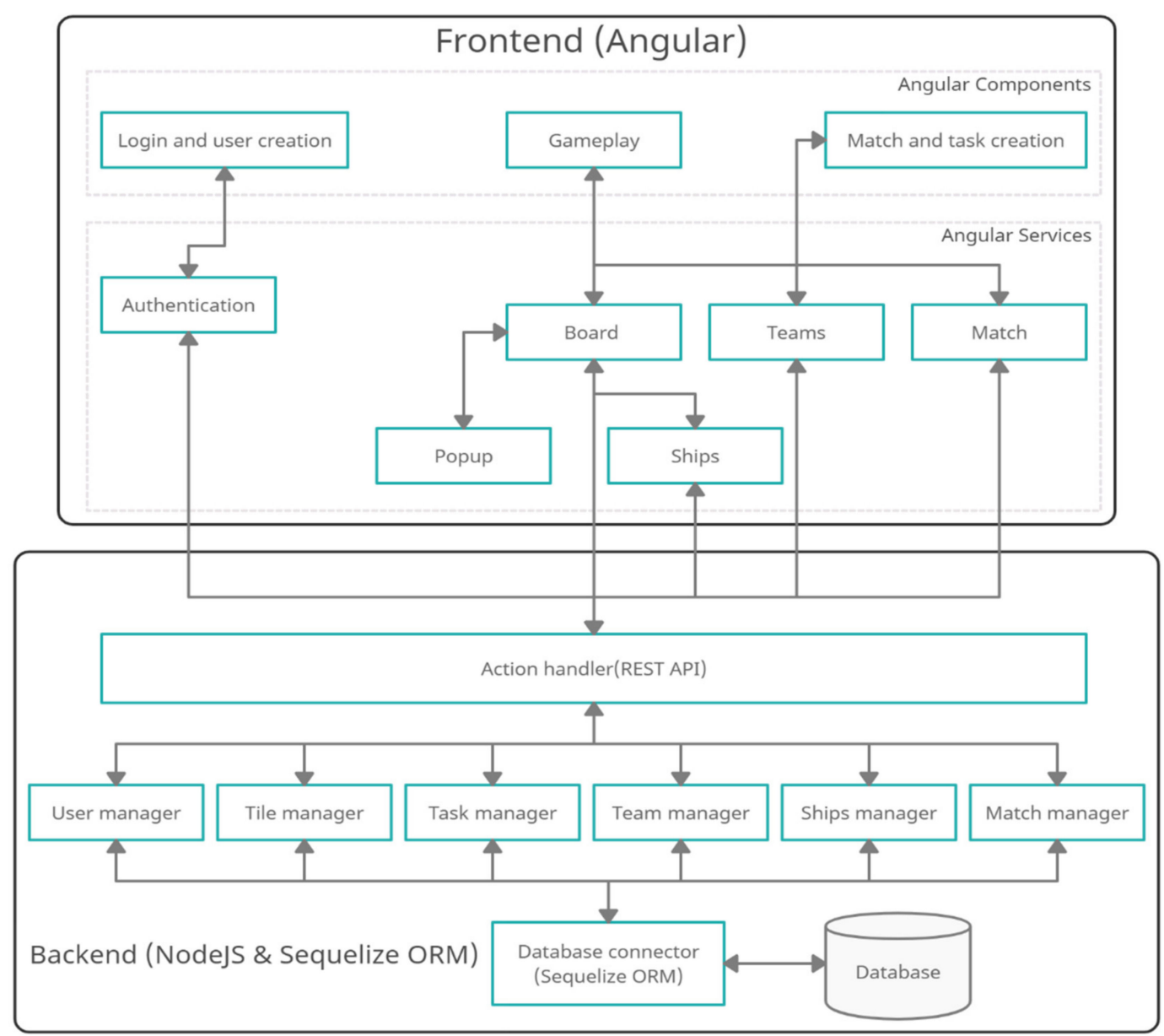

Figure 4. System architecture based on Angular, NodeJS, Sequelize ORM, and MariaDB.

The UI client is only optimized and tested on Google Chrome. The game runs on other browsers and devices, but the game might not be as visually appealing, and some features might not operate properly on other browsers and devices. The frontend UI components are presented to the user with dynamic HTML templates, with data provided by the angular components. The visuals of HTML templates are styled using CSS style sheets.

\subsubsection{Current State of the Game-Low Fidelity Prototype}

The game currently has several features available from the UI for the users. Users can create accounts and $\log$ in to these accounts. The accounts are very simple and only have a username and an ID. The login component simply checks if the username exists in the database. If it does, then the user proceeds to the overview page. The overview page has a list of the matches that the user is in. There is also a navigation bar that has three options: home, create match, and join match. The home button takes the user back to the overview page if the user is not already on the overview page. The join match button opens a field with a six-character join code of numbers and capital letters. Submitting a valid code lets the user join an existing match and one of the two teams. The create match creates a match, a join code, and a lobby for the two teams. The user who created the match has the role of host for that match. A host can proceed from the team lobby and create tasks and then start the match.

When a match has started, all users who are in a team that is in that match will enter the game board if they are still in the lobby; otherwise, they can join from the overview page. The board has $8 \times 8$ tiles, where each tile has a task that should be performed before claiming that tile that could hide a ship. On the board, tiles can be claimed by clicking on an unclaimed tile that will open a popup window with a green button; when clicked, this 
will claim the tile. If the claimed tile hides a ship, then the tile will turn green and play an explosion sound. If not, the tile will turn red and play a splashing sound. The explosion sound will be different depending on if a part of a ship was found or if an entire ship has been found from claiming this tile. Hovering over a tile on the game board will also open a popup window but this cannot be clicked on because it closes on mouse movement.

\section{Testing: Game Testing and Formative Evaluation Method}

\subsection{Playtest-Cool Teknik: Research Context and Participants}

The event "Cool teknik" or in English "Cool tech", is an event in the city of Skellefteå located in northern Sweden where children aged 9-13 years test and learn more about emerging technology. During this event, a group of 13 participants tested Battleships. The participants were 8-11 years and all males. This event had several stations. Due to time restraints, 18 simple physical tasks were prepared in advance with both individual and team tasks. Some examples are "spin around 5 times", "move the door", and "take a lap around the room". Due to the time restraints, technical issues, and the fact that our station consisted of several tests, only one of the two planned groups could test the Battleship-PA game. The group that could not participate in testing at this time were a group of 8 females. The size of the testing group was not ideal according to the classic usability testing guideline: The optimal number for medium-large projects is at least 15, but still large enough for the ideal number of participants for small projects $(n=7)$ [29]. It was impossible to set up more gameplay sessions with school classes, both because this study was conducted over the Swedish summer break and because of the COVID-19 pandemic.

\subsection{Research Method and Data Collection Instruments}

In this mixed-method study comprising of qualitative and quantitative approaches [30], we evaluated the user experience and impact of the Battleship-PA game to promote motivation, cooperation, collaboration, and behavioral changes towards PA among school aged children in an informal setting.

To collect feedback about the game and its impact, a user experience questionnaire was developed comprising four sections on a five-point Likert scale with agreement questions: user experience, motivation, audiovisuals, and impact of physical activity. Questions in all sections were based on questions from studies researched previously [24,31,32]. The user experience is divided into four subsections: general experience, feedback adequacy, presence, and social interaction. Each section also includes an open field question where participants can motivate their answers and freely comment their thoughts about the game. In addition, the user experience section consisted of questions about what the participants thought about playing the game and their interaction with their team. The motivation section assessed what the participants enjoyed about the game, if they did. The audiovisual section gauged what the participants thought about the looks and sounds of the game. The active school transport impact section measured their attitude towards active school transportation and if they learned more about active school transportation by playing the game.

\subsection{Data Collection Procedure}

The testing and data collection took place in August 2021. The participants were divided into four teams and selected one user per team and were helped to set up matches with the 18 prepared tasks. During the game, the participants were observed directly and provided some insights about the game and the playtest process. As we watched the participants, we wrote down each observation in a notebook to avoid missing any data. During direct observation, we were focused on how the participants were physically active during gameplay and observed motivational behaviors related to cooperation, collaboration, competition, and teamplay.

In addition, voice recordings from two of the teams were recorded and then transcribed. After the game had ended the participants anonymously answered a user experience and 
impact questionnaire using Google forms for additional insights about the game and the testing process. They completed a questionnaire on their thoughts about the Battleship-PA game using a Likert scale: 1—strongly disagree and 5-strongly agree. The questionnaire wording was too complex for some participants and needed an explanation to help them answer some questions. Their answers are shown in the next sections.

The user experience and impact questionnaire was presented according to the sections of the user experience constructs derived from [24,31,32], motivation constructs derived from [24], audiovisuals experience constructs derived from [31], and impact on physical activity and active school transport concepts derived from [24].

\subsection{Reliability and Validity}

The user experience and impact questionnaire were first prepared in the English language, administered in the Swedish language, and later translated back to the English language before performing statistical analysis. Since the respondents were children, we ensured that the questionnaire items were designed to be clear, concise, and simple to avoid any confusion. Interaction audio recordings were conducted in Swedish and were transcribed into the English language. Both the translation of questionnaire and transcription of audio recordings were completed by a Swedish native speaker and excellent English speaker based on the Common European Framework of Reference for Languages, often referred to as CEFR. Although open-ended options, such as "other" or "please specify", were provided to the respondents, the reliability of the results could be affected by questions that might have placed a need to satisfy the researchers by respondents answering what is believed to be the accurate answer.

A measure of reliability, Cronbach's alpha on subscales user experience (0.85), motivation (0.88) and perception about physical activity and active school transport (0.74), were relatively high in general [33].

\subsection{Data Analysis Procedure}

The data obtained through the user experience and impact questionnaire were analyzed based on a quantitative research approach using descriptive statistics. The direct observation and audio recording data were analyzed using a qualitative data analysis approach. Working as a team, we collected our observational notes, grouped the observations that refer to common behavior exhibited by the participants, interpreted the observations, and prioritized our findings accordingly. For example, we noted cooperation when team members show signs of agreement to adopt to a particular game strategy.

\subsection{Ethical Consideration}

Conducting research with children requires paying special attention and following due diligence to research ethics. This study was largely guided by the Code of Ethics of the World Medical Association (Declaration of Helsinki), even though this study is not medically inclined and no personal health related data were collected. All data from the event were collected with informed consent from the children's guardians during the event; the children also gave their informed consent to participate. The participants' personal data were anonymized with only ages and genders of their demographic information recorded for research purposes. The study was approved by the regional ethical committee in Umeå (Dr 2018-10-31 M).

\section{Results}

This section focused on the presentation of the results obtained from the analysis of the study data. The results are presented according to analysis of audio recordings, direct observations, and the user experience questionnaire to address the specific research question guiding this study: what is the user experience and impact of the BattleshipPA game to promote motivation for physical activity among school-aged children in an informal setting? 


\subsection{Playtest: Audio Recording and Direct Observation Results}

Team 2 challenged Team 4, resulting in the second match being Team 1 against Team 3; the resolution of both matches is shown in Figure 5. While there were some technical issues, most participants seemed to enjoy the game and both games were able to be played until completion.

\begin{tabular}{|c|c|c|c|c|c|c|c|c|}
\hline \multicolumn{9}{|c|}{$\begin{array}{c}\text { Lag } 2 \text { har vunnit! } \\
\text { Lag } 1 \text { Du } / \text { Pō̄ive: } 13\end{array}$} \\
\hline A. & 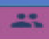 & 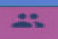 & $\dot{\hbar}$ & $\dot{k}$ & $\dot{x}$ & $\pi$ & $x^{2}$ & $\pi$ \\
\hline $\mathrm{B}$ & 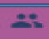 & $x_{i}^{*}$ & $\approx$ & $\bar{k}$ & $x^{*}$ & $x_{1}^{2}$ & $\approx$ & $i=$ \\
\hline c & $\dot{x}=$ & $\hbar$ & $\pi$ & $\because$ & $\pi$ & \{ & 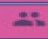 & $\pi=$ \\
\hline $\bar{D}$ & $\approx$ & $\bar{x}=$ & $\approx$ & $\pi$ & $\bar{x}=$ & $\pi$ & $\hbar$ & 7 \\
\hline E & $\approx$ & $\bar{\hbar}$ & $x^{2}$ & $\dot{x}=$ & $\approx$ & $\hbar$ & 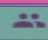 & * \\
\hline $\bar{F}$ & $2 x$ & $\dot{x}=$ & $\hbar$ & $\bar{k}$ & 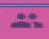 & $x_{1}^{*}$ & $\dot{k}=$ & 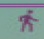 \\
\hline G & $\approx$ & $\bar{\hbar}$ & $\approx$ & $\pi$ & 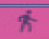 & $\hbar$ & $\approx$ & $\hbar$ \\
\hline \multirow[t]{2}{*}{$\mathrm{H}$} & $\pi$ & 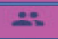 & 5 & क & 4 & $\approx$ & $\bar{x}$ & 4 \\
\hline & 1 & 2 & 3 & 4 & 5 & 6 & 7 & 8 \\
\hline \multicolumn{9}{|c|}{ Lag 2 Motstándare $/ /$ POĀNG: 18} \\
\hline A & $\because$ & $\dot{x}$ & ti & $\pi$ & T & 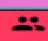 & ti & t \\
\hline B & $\approx$ & $\bar{x}=$ & $\dot{x}=$ & $\dot{k}=$ & 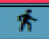 & 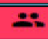 & $\pi$ & $\approx$ \\
\hline $\bar{C}$ & $\dot{x}=$ & $\bar{k}$ & $\ddot{\sim}$ & 政 & $\bar{k}$ & 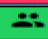 & 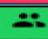 & $\approx$ \\
\hline $\bar{D}$ & $\bar{x}=$ & $\bar{k}=$ & k & $\bar{k}$ & $\bar{x}$ & 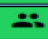 & $k=$ & $\pi$ \\
\hline $\bar{E}$ & $\pi$ & $x_{i}$ & ; & $\bar{k}$ & $\bar{k}$ & 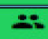 & $\bar{k}$ & $x^{*}$ \\
\hline $\bar{F}$ & $\bar{k}$ & $\hbar$ & ; & 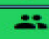 & $\pi$ & * & $\bar{k}=$ & $\because$ \\
\hline $\bar{G}$ & 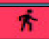 & 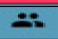 & 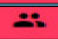 & $\bar{k}$ & $\pi$ & $\pi$ & $\pi$ & $\bar{x}$ \\
\hline \multirow[t]{2}{*}{$\mathrm{H}$} & ; & $\dot{\lambda}=$ & $\hbar$ & $\approx$ & $\hbar$ & 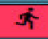 & $\lambda=$ & $x$ \\
\hline & 1 & 2 & 3 & 4 & 5 & 6 & 7 & 8 \\
\hline
\end{tabular}

(a)

\begin{tabular}{|c|c|c|c|c|c|c|c|c|}
\hline \multicolumn{9}{|c|}{$\begin{array}{l}\text { Lag } 2 \text { har vunnit! } \\
\text { Lag } 1 \text { De / POĀXG: } 15\end{array}$} \\
\hline A & $\approx$ & $\not$ & $\approx$ & $x^{*}$ & 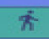 & $\pi$ & $\therefore$ & $\therefore$ \\
\hline B & $s^{2}$ & $\hbar^{2}$ & 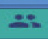 & $\dot{x}$ & $x^{2}$ & $\pi$ & 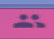 & $\hat{k}=$ \\
\hline $\bar{c}$ & $\hbar$ & $\pi$ & $\bar{k}$ & $x^{2}$ & $\Rightarrow$ & $5^{2}$ & 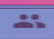 & $x^{2}=$ \\
\hline $\mathrm{D}$ & $\approx$ & $\pi^{2}$ & $\approx$ & $\pi=$ & $\pi$ & $\pi$ & 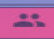 & $\pi^{2}$ \\
\hline $\bar{E}$ & 7 & $\pi$ & $\because$ & $\approx$ & $\pi$ & 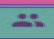 & 4 & $\pi$ \\
\hline$F$ & $\pi$ & $\pi$ & $\bar{\pi}$ & $\approx$ & $\bar{\hbar}$ & $\pi=$ & 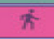 & 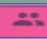 \\
\hline$G$ & $\Delta$ & $\approx$ & $\bar{k}$ & $\pi$ & $\approx$ & $\pi$ & $\not{\hbar}$ & $x^{2}$ \\
\hline $\mathrm{H}$ & $s^{2}$ & $\Rightarrow$ & $\because$ & $\pi$ & $\approx$ & $\pi$ & +4 & $\pi$ \\
\hline & 1 & 2 & 3 & 4 & 5 & 6 & 7 & $\mathrm{~s}$ \\
\hline \multicolumn{9}{|c|}{ Lag 2 Motstindare of POĀNG: $1 \mathrm{~s}$} \\
\hline A & $s^{*}$ & $\pi$ & $\pi$ & $\pi$ & $\bar{k}=$ & $\because$ & $x=$ & $a_{1}^{*}$ \\
\hline B & $\bar{\hbar}$ & T) & $\pi$ & $\bar{k}=$ & $x_{1}^{*}$ & $\pi$ & $\pi$ & $\approx$ \\
\hline C & 7 & $\because$ & $S^{*}$ & 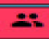 & $\hbar$ & $\pi^{2}$ & $\bar{k}$ & $x_{1}^{2}$ \\
\hline $\mathrm{D}$ & $F^{*}$ & 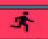 & t & 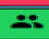 & $\hbar$ & $\bar{k}$ & $\dot{x}=$ & 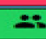 \\
\hline E & 7 & 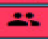 & $\bar{k}$ & $\dot{x}$ & $\approx$ & $\bar{\pi}$ & $\dot{x}=$ & $x^{2}$ \\
\hline $\bar{F}$ & $s^{*}$ & $\bar{\hbar}$ & $\bar{\pi}$ & $\bar{x}=$ & $\pi$ & $\pi=$ & $x^{*}$ & $\bar{k}$ \\
\hline G & $s^{*}$ & $x=$ & $x^{2}$ & $x_{1}^{2}$ & $x_{i}^{*}$ & $\pi$ & $\pi$ & 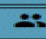 \\
\hline \multirow[t]{2}{*}{$\mathbf{H}$} & 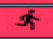 & $\pi$ & $\Longrightarrow$ & $T^{*}$ & $\pi$ & 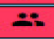 & $\pi$ & $\approx$ \\
\hline & 1 & 2 & 3 & 4 & 5 & 6 & 7 & $\mathrm{~s}$ \\
\hline
\end{tabular}

(b)

Figure 5. (a) Finished match of Team 1 vs. Team 3. Top message: Team 2 has won! (b) Finished match of Team 2 vs. Team 4 . Top message: Team 2 has won!

Similar observations to the audio recordings were made by directly observing the participants during the event. Participants were cooperative within their team. There were elements of competition as teams played against the other team. Some were confused by the visuals, disappointed by the diversity of the tasks and experienced several crashes during testing gameplay. Unlike the recordings, however, some participants enjoyed moving around at the start and became tired at the end of the session. Some participants 
also wished to have their own user to play with instead of sharing one with their team. In addition, participants that had not already played the classical version of Battleships were confused about the objectives and concept of the game.

\subsection{User Experience}

Table 2 presents the descriptive statistics of the opinions of the respondents regarding their experience after playing Battleship-PA. Most respondents strongly agreed with the general user experience statements. For example, when asked about their opinion on whether it was easy to understand how the game worked (1), most of the participants agreed $(\mu=4.15, \sigma=0.89)$. Similarly, most of the participants indicated that they were happy with the game as a whole $(3)(\mu=4.46, \sigma=0.77)$ and would play the game again $(4)$ $(\mu=3.92, \sigma=1.25)$.

Table 2. General user experience response.

\begin{tabular}{cccc}
\hline $\mathbf{S} / \mathbf{N}$ & Statements & $\boldsymbol{\mu}$ & $\boldsymbol{\sigma}$ \\
\hline 1 & It was easy to understand how the game worked & 4.15 & 0.89 \\
\hline 2 & It was clear how close my team was to winning & 4.38 & 0.86 \\
\hline 3 & I am happy with the game as a whole & 4.46 & 0.77 \\
\hline 4 & I would like to play the game again & 3.92 & 1.25 \\
\hline
\end{tabular}

Participants' opinions about the Battleship-PA game are presented in Table 3. The participants mostly agreed that their actions had immediate feedback $(5)(\mu=4.07, \sigma=0.75)$ and that completing tasks felt good (6) $(\mu=4.61, \sigma=0.65)$.

Table 3. Perceived feedback adequacy response.

\begin{tabular}{cccc}
\hline $\mathbf{S} / \mathbf{N}$ & Statements & $\boldsymbol{\mu}$ & $\boldsymbol{\sigma}$ \\
\hline 5 & I received immediate feedback on my actions & 4.07 & 0.75 \\
\hline 6 & It felt good to complete tasks in the game & 4.61 & 0.65 \\
\hline
\end{tabular}

Table 4 describes the participants' immersion when playing the game. Most felt that they concentrated on the game while playing (7) $(\mu=4.23, \sigma=0.83)$, which seems on point with direct observations and the interaction audio recordings. Most participants felt that they had no opinion or did not know if they lost track of time while playing the game (8) $(\mu=3.61, \sigma=0.96)$.

Table 4. Presence response.

\begin{tabular}{cccc}
\hline $\mathbf{S} / \mathbf{N}$ & Statements & $\boldsymbol{\mu}$ & $\boldsymbol{\sigma}$ \\
\hline 7 & I was very focused on the game when I played & 4.23 & 0.83 \\
\hline 8 & I forgot the time while playing the game & 3.61 & 0.96 \\
\hline
\end{tabular}

By analyzing Table 5, some insights about the social aspects of the game could be found. Most participants strongly agreed they were able to interact $(10)(\mu=4.46, \sigma=0.66)$, and have fun with their team $(11)(\mu=4.53, \sigma=0.51)$. Most participants also felt that the game promoted cooperation and competition $(9)(\mu=4.23, \sigma=0.72)$. 
Table 5. Social interaction response.

\begin{tabular}{cccc}
\hline $\mathbf{S} / \mathbf{N}$ & Statements & $\boldsymbol{\mu}$ & $\boldsymbol{\sigma}$ \\
\hline 9 & The game promotes cooperation and/or competition among \\
the players & 4.23 & 0.72 \\
\hline 10 & I was able to interact with other players during the game & 4.46 & 0.66 \\
\hline 11 & I had fun with the other players & 4.53 & 0.51 \\
\hline
\end{tabular}

\subsection{Motivation}

This section measures which aspects of the game motivated the users to play the game (Table 6). The motivational statements that focused on competition, collaboratively discussing tactics within their team, and performing physical activity during gameplay received the strongest agreement from the participants. Particularly, the participants strongly agreed that they wanted to play the game because they were able to compete (12) $(\mu=4.30, \sigma=0.75)$, they liked the opportunity to discuss and plan how their team would play, (15) ( $\mu=4.30, \sigma=0.85)$, it is good for the body to move (17) $(\mu=4.30, \sigma=1.03)$, and to collaborate with other players (13) $(\mu=4.15, \sigma=0.68)$. Most agreed that cooperation, the in-game tasks, and the fact that this was part of their "cool technology" event were motivating factors to play the game. Only one team had audio while playing so it is not surprising that a majority did not know if notifications were a motivational factor because sound was the only notification available in this low fidelity version of the game.

Table 6. Motivation response: I wanted to play the game because ... .

\begin{tabular}{cccc}
\hline $\mathbf{S} / \mathbf{N}$ & Statements & $\mu$ & $\boldsymbol{\sigma}$ \\
\hline 12 & $\ldots$. we got to compete & 4.30 & 0.75 \\
\hline 13 & $\ldots$ of the collaboration with other players & 4.15 & 0.68 \\
\hline 14 & $\ldots$ of the details of the game & 4.07 & 0.95 \\
\hline 15 & $\ldots$ I liked the opportunity to discuss and plan how my team & 4.30 & 0.85 \\
\hline 16 & would play & 3.69 & 0.85 \\
\hline 17 & $\ldots$ of notes in the game (for example, sound effects) & 4.30 & 1.03 \\
\hline 18 & $\ldots$ it is good for the body to move & 3.76 & 0.92 \\
\hline
\end{tabular}

\subsection{Audiovisuals}

This section analyzed participants' thoughts about the audio and visuals. As mentioned in the previous section, only one team had audio and therefore most participants did not know if they enjoyed the sound effects and if it enhanced their experience. However, the team that did have audio seemed to strongly agree that the audio was enjoyable and improved their experience. As for visuals in Table 7, most agreed that the game was visually appealing $(21)(\mu=3.84, \sigma=0.68)$ and fit the mood of the game $(22)(\mu=4.38, \sigma=0.65)$.

\subsection{Impact on Physical Activity and Active School Transport Concepts}

Finally, this section measured what the participants thought about the impact of physical activity in the game. In Table 8, most participants agreed that the game helped them be physically active $(23)(\mu=3.46, \sigma=1.05)$ and strongly agreed that they liked performing physical activities in the game $(24)(\mu=4.07, \sigma=1.18)$. 
Table 7. Perceived audiovisual adequacy response: What do you think of these claims?

\begin{tabular}{cccc}
\hline $\mathbf{S} / \mathbf{N}$ & Statements & $\boldsymbol{\mu}$ & $\boldsymbol{\sigma}$ \\
\hline 19 & I liked the sound effects in the game & 3.46 & 0.87 \\
\hline 20 & The game's sound effects improved my gaming experience & 3.38 & 0.96 \\
\hline 21 & I think the game was visually appealing & 3.84 & 0.68 \\
\hline 22 & I think the game's appearance matched the game's theme & 4.38 & 0.65 \\
\hline
\end{tabular}

Table 8. Perception about physical activity/active school transport response: What do you think of these claims?

\begin{tabular}{cccc}
\hline $\mathbf{S} / \mathbf{N}$ & Statements & $\boldsymbol{\mu}$ & $\boldsymbol{\sigma}$ \\
\hline 23 & The game helped me be physically active & 3.46 & 1.05 \\
\hline 24 & I enjoyed performing physical activities in the game & 4.07 & 1.18 \\
\hline
\end{tabular}

\section{Discussion}

There were several evaluations of the gameplay session: participants' interaction audio recordings, the observations, and the questionnaire. The audio recordings revealed that the participants were highly competitive and invested in the result of the match. This highlights the importance of competition as a motivating factor [9]. However, several participants were frustrated or unmotivated by the fact that several tasks had to be repeated multiple times. Repetition could be an issue that decreases users' interest and investment in the game. Therefore, it is important to consider what aspects of the game felt repetitive and how to avoid/change them or add incentives to motivate the aspects that feel repetitive [9] to keep users engaged in the game. Nevertheless, the game is intended to be used in a school setting, and the notion of fun and engagement could be negotiated. Prior work [34] shows that student engagement is built on behavioral engagement, i.e., participation and involvement, emotional engagement in the form of positive and negative reactions, as well as cognitive engagement, i.e., level of investment in learning.

Others [35] found that learning is fun when there are choices, relevance, active learning, and camaraderie. This was achieved here by developing the tasks of the game that challenged the children physically, emotionally, and cognitively, as well as inspiring collaborative aspects. This approach allows teachers to create the task in relation to the curriculum. This enhances the possibility that activity and learning will occur and could encourage schools to use the game.

Rutberg and Lindqvist [26] found that teacher's involvement in designing assignments during active school transport enhanced student engagement in using active transport as well as enhancing teachers' engagement in participating in the intervention. The recordings also highlighted that the visuals were confusing, and the rules of the game were unclear to some participants. While there was not as much frustration as for the repetition problem, some participants clearly struggled to comprehend the visuals and felt somewhat unmotivated to keep playing. Therefore, having clear progression and rules of a game is essential for motivating users to keep user engagement and avoid frustrations when playing [9]. From observing the participants during the event, in addition to participants being competitive, they seemed to enjoy the collaborative aspect of the game, which is also supported by the questionnaire. This signifies the importance of collaboration as a motivation for gamification [9,24], which is also important in behavioral change [36]. In Table 9, we used the eight core Octalysis framework to highlight the required improvement in the future version of the game. 
Table 9. The eight core drives of gamification of Octalysis in the current version of the game and improvement ideas based on the eight drives.

\begin{tabular}{|c|c|c|}
\hline Core Drives & In Battleships & Gamification Improvement Ideas \\
\hline Accomplishment & Completing tasks or finding ships & Experience points and leveling system. \\
\hline Avoidance & Avoid losing & $\begin{array}{l}\text { Losing experience or game currency by using } \\
\text { motorized transport. }\end{array}$ \\
\hline Empowerment & Not in this version & $\begin{array}{l}\text { Allow users to upload pictures to use for } \\
\text { profile pictures. }\end{array}$ \\
\hline Epic meaning & Not in this version & Benefits from physical activity. \\
\hline Ownership & Not in this version & $\begin{array}{l}\text { Individual avatar with items that can be seen by } \\
\text { other users. }\end{array}$ \\
\hline Scarcity & Not in this version & $\begin{array}{l}\text { Set a limit on avatar items, could be time } \\
\text { availability or a set amount of each item. }\end{array}$ \\
\hline Social influence & $\begin{array}{l}\text { Collaboration with team, competition } \\
\text { against opponents }\end{array}$ & $\begin{array}{l}\text { Chat with other users, add other users to friend } \\
\text { list and share achievements or avatars to others. }\end{array}$ \\
\hline Unpredictability & Not knowing which tiles are ships & Adding secrets behind some tiles \\
\hline
\end{tabular}

The results of the user experience aspects were mostly positive, as the respondents mostly agreed with the general user experience constructs, such as the game being easy to understand, being happy with the game and would like to play the game again. These user experience results were similar to the outcome of an earlier study [21], in which the students reported a highly positive experience using games as interventions for increased physical activity. In addition, most of the students responded to a positive impact from motivational statements that focused on competition, collaboration within their teams, and performing physical activity during gameplay, as reported in earlier studies $[15,18,20,24]$.

While the game is playable, there are several features that would drastically improve the game. Several points of the Octalysis framework [12] recommend adding features that are customizable for all users and are owned by a single user. We could provide users with their own customizable avatars and add purchasable items for those avatars. Studies have shown that player-avatar identification led to increased motivation, time spent in gameplay, and completed game levels [37,38]. These items were bought with game currency gained by playing the game. This increases motivations for playing the game. Another feature motivating continuous play could be to add a progression system and achievements. For example, adding individual levels for users that grow with experience points. This could change by interacting with the game. Certain levels or events would grant the user an achievement or milestone. There could also be extra things hidden under some of the tiles, e.g., extra experience points or a cosmetic item for avatars. This adds an element of surprise for users and might motivate users to perform tasks even if they think that they are repetitive [9]. Moreover, adding these motivating features will minimize novelty effect, perceived enjoyment, and usefulness of gamification diminishing with use [20].

Another feature that also would improve motivation would be to add a social system for users. Communication was not a problem during the game tests because all participants were in the same room; thus, it might become an issue when the users are separated. Since each board has 64 tiles, it might be challenging for hosts to come up with 64 unique tasks. Thus, adding a feature to add common tasks, such as "walking to school" or "biking to school", would probably simplify the match creation process. To show hosts how the icons would look on the board when creating tasks, the current dropdown menu with text could be replaced with a menu showing the icons visually. More icons could also be added to divide tasks more easily into different categories; thus, users would more easily understand the task on each tile. The method to choose which tasks are selected could also 
be improved so that each task does not repeat before all created tasks have been selected. This makes the tasks feel less repetitive.

While users might simply enjoy doing tasks and playing the game, it currently does not teach them anything about the benefits of daily physical activity. Hence, tools to teach users about these benefits and keep track of their current progress and put that into perspective might motivate users to continue playing. For example, keeping track of how much energy a team or user has used while playing the game and comparing how long you could run with that energy or what environmental impact the user or team has had by not using motorized transport.

The audio and visuals of the game could also be improved. We noted several confusions regarding the visuals from the observations and the audio recordings. Two of the confusions were that users were unsure about their score and which of the two boards belonged to their team. It was also unclear when one team had won the match; some participants were unsure of the rules of the game. Therefore, the visuals could be updated to clarify these aspects and add a way to see how many ships there are left for both teams. Additionally, a tutorial for users unfamiliar with the classic game could be added so that all users can understand the rules of the game. Another way to clear some of these confusions could be to add feedback features that clearly communicate with the user when something is performed incorrectly, or something has gone wrong.

This study has significant value but also several limitations. First, the group size of 13 people is more than the recommended amount for small user tests [29], but it is also too small to draw any general conclusions about the game. Second, the lack of gender diversity in the testing group makes the results of the study less generalizable. Third, all the participants are from Skelleftea in northern Sweden and around the same age-further testing with participants of different ages and from different countries or other parts of the country would be needed before any general conclusions can be made.

\section{Conclusions and Future Work}

In conclusion, the web-based game Battleships-PA was presented with its conceptual and technical details. The game was designed with inspiration from the game Tic-TacTraining. The main purpose of this study was to present the entire game development process of a PA game within a non-school context and gain insights from the gameplay session on how the game could be improved. By analyzing the answers from the questionnaire, the audio recordings, and observing the participants during the session, we concluded that the users enjoyed the collaborative and competitive aspects of the game but were confused by the visuals of the game. The results indicated their opinion regarding the game to improve motivation to be physically active in non-school settings. Children enjoyed collaborating and competing with others as well as completing tasks. However, the Octalysis framework suggested several features that could improve the game with a focus on long-term user involvement and an increase in clarity of the game visuals. Moreover, it is important to have clear visuals and rules or a tutorial so that all users can understand the game and thereby reducing the possibility of the novelty effect. The results have potential to impact future gamified PA interventions or solutions. The school setting is overwhelmed with tasks, and we wanted to create a game that has the potential to facilitate learning linked to the curriculum that was easy to use. Therefore, this game might also be interesting to researchers or teachers who wish to implement learning through gamification and simultaneously encourage PA in school classrooms. The architecture of the game might also be useful to developers who want to create web-based cooperative multiplayer games.

Future work involves several features that would improve the game and that can be implemented as suggested in the discussion section. Additional testing would be required to gain more accurate data on the motivational aspects of the game and if it motivates PA. This version of the game is still a competition between two teams; therefore, the next developmental phase of the game would focus on tweaking the game board to accommodate and be played by only one team to encourage collaboration versus competition. 
Additionally, observing how different age groups and more diverse gender distributions would react to the game could help determine the best target groups of children and ages. Lastly, our future research can use the knowledge gained in this study to develop a framework that integrates gamification in persuasive technologies for active school transport. In order to improve physical activity for children and adolescents, promoting and motivating them to use active transportation daily has been shown to be an effective way to increase physical activity [39]. While there are numerous benefits of AT as a tool to increase physical activity, only $57 \%$ of schoolchildren of the ages 6-15 use AT to school during spring and fall in Sweden. Only 47\% use AT during the winter [40]. Even globally, there is a decrease in AT for children and adolescents, which will likely continue unless interventions are made [41,42]. This future research would optimally focus on ways to increase AST among children in Sweden. Methods of travel between home and school using non-motorized means could be studied to determine if the game can motivate long-term usage of active school transport.

Author Contributions: Conceptualization, S.S.O., R.B., T.H.L. and S.M.B.; methodology, S.S.O., R.B., T.H.L. and S.M.B.; software, S.S.O., T.H.L. and S.M.B.; validation, A.-K.L., R.B., S.R. and S.S.O.; formal analysis, S.S.O.; investigation, S.S.O.; resources, A.-K.L., R.B., T.H.L. and S.R.; data curation, S.S.O. and S.M.B.; writing-original draft preparation, S.S.O., T.H.L. and S.M.B.; writing-review and editing, S.S.O., R.B., A.-K.L., S.R. and S.M.B.; visualization, S.M.B.; supervision, S.S.O.; project administration, A.-K.L., R.B., T.H.L. and S.R.; funding acquisition, A.-K.L. and S.R. All authors have read and agreed to the published version of the manuscript.

Funding: This research was funded by Vinnova, grant number 2020-01867.

Institutional Review Board Statement: Not applicable.

Informed Consent Statement: Informed consent was obtained from all guardians of subjects involved in the study.

Data Availability Statement: Not appliable.

Conflicts of Interest: The authors declare that they have no known competing financial interests or personal relationships that could have appeared to influence the work reported in this paper.

\section{References}

1. World Health Organization. Global Recommendations on Physical Activity for Health; World Health Organization: Geneva, Switzerland, 2010.

2. Guthold, R.; Stevens, G.A.; Riley, L.M.; Bull, F.C. Global trends in insufficient physical activity among adolescents: A pooled analysis of 298 population-based surveys with 1.6 million participants. Lancet Child Adolesc. Health 2020, 4, 23-35. [CrossRef]

3. Oja, P.; Titze, S.; Bauman, A.; de Geus, B.; Krenn, P.; Reger-Nash, B.; Kohlberger, T. Health benefits of cycling: A systematic review: Cycling and health. Scand. J. Med. Sci. Sports 2011, 21, 496-509. [CrossRef] [PubMed]

4. Gtschi, T.; Garrard, J.; Giles-Corti, B. Cycling as a part of daily life: A review of health perspectives. Transp. Rev. 2016, 36, 45-71. [CrossRef]

5. Ding, D.; Lawson, K.D.; Kolbe-Alexander, T.L.; Finkelstein, E.A.; Katzmarzyk, P.T.; van Mechelen, W.; Pratt, M. The economic burden of physical inactivity: A global analysis of major non-communicable diseases. Lancet 2016, 388, 1311-1324. [CrossRef]

6. Australian Government Department of Health. Australian 24-Hour Movement Guidelines for Children (5-12 years) and Young People (13-17 years): An Integration of Physical Activity, Sedentary Behaviour, and Sleep; Australian Government Department of Health: Canberra, Australia, 2019.

7. Telama, R.; Yang, X.; Leskinen, E.; Kankaanpää, A.; Hirvensalo, M.; Tammelin, T.; Viikari, J.S.A.; Raitakari, O.T. Tracking of physical activity from early childhood through youth into adulthood. Med. Sci. Sports Exerc. 2014, 46, 955-962. [CrossRef]

8. World Health Organization. More Active People for a Healthier World: Global Action Plan on Physical Activity 2018-2030; World Health Organization: Geneva, Switzerland, 2018; ISBN 978-92-4-151418-7.

9. Laine, T.H.; Lindberg, R.S.N. Designing engaging games for education: A systematic literature review on game motivators and design principles. IEEE Trans. Learn. Technol. 2020, 13, 804-821. [CrossRef]

10. Lindqvist, A.-K.; Löf, M.; Ek, A.; Rutberg, S. Active school transportation in winter conditions: Biking together is warmer. Int. J. Environ. Res. Public Health 2019, 16, 234. [CrossRef]

11. Kazhamiakin, R.; Marconi, A.; Perillo, M.; Pistore, M.; Valetto, G.; Piras, L.; Avesani, F.; Perri, N. Using gamification to incentivize sustainable urban mobility. In Proceedings of the 2015 IEEE First International Smart Cities Conference (ISC2), Guadalajara, Mexico, 25-28 October 2015; pp. 1-6. 
12. Chou, Y. Actionable Gamification: Beyond Points, Badges, and Leaderboards; Octalysis Media: Milpitas, CA, USA, 2015.

13. Hunicke, R.; LeBlanc, M.; Zubek, R. MDA: A Formal Approach to Game Design and Game Research. In Proceedings of the AAAI Workshop on Challenges in Game AI; AAAI: San Jose, CA, USA, 2004; p. 6.

14. Marczewski, A. Even Ninja Monkeys Like to Play: Gamification, Game Thinking \& Motivational Design; Gamified: Addlestone, UK, 2015; ISBN 978-1-364-95552-6.

15. Patricio, D.S.; Aires, Y.R.; Medeiros, C.M. Gamification: A tool to increase the frequency of physical activity in overweight adolescents. J. Exerc. Physiol. Online 2020, 23, 13-24.

16. Corepal, R.; Best, P.; O’Neill, R.; Kee, F.; Badham, J.; Dunne, L.; Miller, S.; Connolly, P.; Cupples, M.E.; van Sluijs, E.M.F.; et al. A feasibility study of 'The StepSmart Challenge' to promote physical activity in adolescents. Pilot Feasibility Stud. 2019, 5, 1-15. [CrossRef]

17. Lister, C.; West, J.H.; Cannon, B.; Sax, T.; Brodegard, D. Just a Fad? Gamification in Health and Fitness Apps. JMIR Serious Games 2014, 2, e9. [CrossRef]

18. Kappen, D.L.; Mirza-Babaei, P.; Nacke, L.E. Gamification through the Application of Motivational Affordances for Physical Activity Technology. In Proceedings of the Annual Symposium on Computer-Human Interaction in Play, Amsterdam, The Netherlands, 15-18 October 2017; pp. 5-18. [CrossRef]

19. Zuckerman, O.; Gal-Oz, A. Deconstructing gamification: Evaluating the effectiveness of continuous measurement, virtual rewards, and social comparison for promoting physical activity. Pers. Ubiquitous Comput. 2014, 18, 1705-1719. [CrossRef]

20. Koivisto, J.; Hamari, J. Demographic differences in perceived benefits from gamification. Comput. Hum. Behav. 2014, 35, 179-188. [CrossRef]

21. González, C.S.; Gómez, N.; Navarro, V.; Cairós, M.; Quirce, C.; Toledo, P.; Marrero-Gordillo, N. Learning healthy lifestyles through active videogames, motor games and the gamification of educational activities. Comput. Hum. Behav. 2016, 55, 529-551. [CrossRef]

22. Hunter, R.F.; de Silva, D.; Reynolds, V.; Bird, W.; Fox, K.R. International inter-school competition to encourage children to walk to school: A mixed methods feasibility study. BMC Res. Notes 2015, 8, 19. [CrossRef]

23. Julien, C.; Castelli, D.; Bray, D.; Lee, S.; Burson, S.; Jung, Y. Project SMART: A cooperative educational game to increase physical activity in elementary schools. Smart Health 2020, 19, 100163. [CrossRef]

24. Laine, T.H.; Normark, J.; Lindvall, H.; Lindqvist, A.-K.; Rutberg, S. A distributed multiplayer game to promote active transport at workplaces: User-centred design, implementation and lessons learned. IEEE Trans. Games 2020, 12, 386-397. [CrossRef]

25. Lindqvist, A.; Castelli, D.; Hallberg, J.; Rutberg, S. The Praise and Price of Pokémon GO: A Qualitative Study of Children's and Parents' Experiences. JMIR Serious Games 2018, 6, e1. [CrossRef]

26. Rutberg, S.; Lindqvist, A.-K. Active school transportation is an investment in school Health. Health Behav. Policy Rev. 2018, 5 , 88-97. [CrossRef]

27. Lindqvist, A.; Rutberg, S. One step forward: The development of a program promoting active school transportation. JMIR Res. Protoc. 2018, 7, e123. [CrossRef]

28. Ramadan, R.; Widyani, Y. Game development life cycle guidelines. In Proceedings of the International Conference on Advanced Computer Science and Information Systems, Kuta, Indonesia, 28-29 September 2013; pp. 95-100. [CrossRef]

29. Nielsen, J.; Landauer, T.K. A mathematical model of the finding of usability problems. In Proceedings of the INTERACT ' 93 and CHI '93 Conference on Human Factors in Computing Systems (CHI '93), Amsterdam, The Netherlands, 24-29 May 1993; pp. 206-213. [CrossRef]

30. Creswell, J.W.; Clark, V.L.P. Designing and Conducting Mixed Methods Research, 3rd ed.; SAGE Publications: London, UK, 2017.

31. Fokides, E.; Atsikpasi, P.; Kaimara, P.; Deliyannis, I. Let players evaluate serious games. Design and validation of the serious games evaluation scale. ICGA J. 2019, 41, 116-137. [CrossRef]

32. Petri, G.; von Wangenheim, C.G.; Borgatto, A.F. A Large-Scale Evaluation of a Model for the Evaluation of Games for Teaching Software Engineering. In Proceedings of the 2017 IEEE/ACM 39th International Conference on Software Engineering: Software Engineering Education and Training Track (ICSE-SEET), Buenos Aires, Argentina, 20-28 May 2017; pp. 180-189. [CrossRef]

33. Taber, K.S. The Use of Cronbach's Alpha When Developing and Reporting Research Instruments in Science Education. Res. Sci. Educ. 2018, 48, 1273-1296. [CrossRef]

34. Fredricks, J.A.; Blumenfeld, P.C.; Paris, A.H. School engagement: Potential of the concept, state of the evidence. Rev. Educ. Res. 2004, 74, 59-109. [CrossRef]

35. Zinn, W.-O.R. Making fun of school, or why does learning have to be such a drag? Six key elements for motivating learning. Int. J. Learn. 2008, 15, 153-160.

36. Savolainen, E.; Rutberg, S.; Backman, Y.; Lindqvist, A.-K. Long-Term Perspectives of a School-Based Intervention to Promote Active School Transportation. Int. J. Environ. Res. Public Health 2020, 17, 5006. [CrossRef]

37. Kao, D.; Harrell, D.F. The Effects of Badges and Avatar Identification on Play and Making in Educational Games. In Proceedings of the 2018 CHI Conference on Human Factors in Computing Systems, Montreal, QC, Canada, 21-26 April 2018; pp. 1-19. [CrossRef]

38. Wang, X.; Butt, A.H.; Zhang, Q.; Shafique, M.N.; Ahmad, H.; Nawaz, Z. Gaming avatar can influence sustainable healthy lifestyle: Be like an avatar. Sustainability 2020, 12, 1998. [CrossRef] 
39. Williams, G.C.; Borghese, M.M.; Janssen, I. Neighborhood walkability and objectively measured active transportation among 10 to 13-year-olds. J. Transp. Health 2018, 8, 202-209. [CrossRef]

40. Nyström, C.D.; Larsson, C.; Ehrenblad, B.; Eneroth, H.; Eriksson, U.; Friberg, M.; Hagströmer, M.; Lindroos, A.K.; Reilly, J.J.; Löf, M. Results from Sweden's 2016 report card on physical activity for children and youth. J. Phys. Act. Health 2016, 13, S284-S290. [CrossRef]

41. Johansson, C.; Lövenheim, B.; Schantz, P.; Wahlgren, L.; Almström, P.; Markstedt, A.; Strömgren, M.; Forsberg, B.; Sommar, J.N. Impacts on air pollution and health by changing commuting from car to bicycle. Sci. Total Environ. 2017, 584-585, 55-63. [CrossRef]

42. Johansson, K.; Laflamme, L.; Hasselberg, M. Active commuting to and from school among Swedish children-A national and regional study. Eur. J. Public Health 2011, 22, 209-214. [CrossRef] 